\title{
SECULAR PERTURBATIONS NEAR MEAN MOTION RESONANCES
}

\author{
A. A. CHRISTOU \\ Astronomy Unit, Queen Mary \& Westfield College, London E1 4NS, United Kingdom, \\ E-mail:A.Christou@qmw.ac.uk
}

In this work we attempt to make progress into assessing the importance of secular interactions between planetary satellites. In recent years, discrepancies have been observed in the expected positions of small planetary satellites (Bosh \& Rivkin, 1996; Roddier et al., 1998). The existing ephemerides-producing algorithms for these objects assume fixed, elliptical and inclined orbits whose rate of precession is determined by oblateness alone. Even though the masses of these satellites are quite small relative to the planet $\left(\sim 10^{-9}-10^{-10}\right)$ their small mutual separations and the existence of much larger satellites further out leaves open the possibility that in some cases at least the fixed-orbit assumption is only a crude approximation to reality. Two important dynamical mechanisms through which these orbits may evolve are resonant or secular interactions. In order to explore the possibility of the latter we have set up a simple planar system where an satellite in a circular orbit around a spherical planet is perturbing a massless particle which moves in proximity to various mean motion resonances. We aim to examine the effect of the resonance on the particle's reference orbit by measuring the secular frequency. The effects of oblateness have not been taken into account as they are adequately modeled by orbit-fitting theories and can thus be readily subtracted.

\section{TABLE I}

Initial conditions for the numerical integrations near the 2:1 resonance (upper row) and the $1: 1$ resonance (lower row). The symbol $\Delta \alpha$ denotes the resolution of the sampling in the initial value of $\alpha$ and $e$ the initial eccentricity.

\begin{tabular}{lrrr}
\hline Range in $\alpha$ & $\Delta \alpha$ & $e$ & Samples \\
\hline $0.58-0.68$ & 0.005 & 0.05 & $2 \times 21$ \\
$0.80-0.90$ & 0.002 & 0.001 & 51 \\
\hline
\end{tabular}

We have integrated the motion of a particle for a range of values of the semimajor axis $\alpha$ (Table I) using the SWIFT_RMVS2 symplectic code (Levison \& Duncan, 1994). The output was reduced to a list of frequencies, amplitudes and phases using a program based on the Frequency Modified Fourier Transform algorithm (Šidlichovský \& Nesvorný , 1997). We then compare the resulting frequencies with analytical estimates derived with a second order secular theory (Christou, 1998).

In this manner we have investigated (a) the vicinity of the 2:1 resonance (b) orbital evolution in proximity to the planet (Christou \& Murray, 1998). Our results can be summarized in Table II where we show numerical and analytical estimates 
of the secular frequency for values of the semi-major axis which were chosen to be close to specific first order $(p+1: p)$ mean motion resonance locations.

\section{TABLE II}

Estimates of the secular frequency for values of the semimajor axis close to specific first order mean motion resonance locations. The suffixes (A), (P) give the initial position of the particle relative to the satellite (pericentric or apocentric conjunction). For all cases apart from the first two rows the particle was tested with a pericentric conjuction start only. Units used are rad per perturber revolution.

\begin{tabular}{lrrrr}
\hline$\alpha$ & Resonance & Order 1 Est. & Order 2 Est. & Numer. Est. \\
\hline 0.610 & $2: 1(\mathrm{P})$ & 0.00540443 & 0.00931030 & 0.00933683 \\
0.610 & $2: 1(\mathrm{~A})$ & 0.00537956 & 0.00899243 & 0.00914993 \\
0.824 & $4: 3$ & 0.000309033 & 0.00104631 & 0.000996402 \\
0.860 & $5: 4$ & 0.000493989 & 0.00134269 & 0.00128978 \\
0.883 & $6: 5$ & 0.000721684 & 0.00210074 & 0.00201287 \\
0.900 & $1: 1$ & 0.000969363 & 0.00196977 & 0.00198843 \\
\hline
\end{tabular}

We clearly see that the Laplace-Lagrange approximation (third column) is insufficient for describing the secular evolution of the particle evolving in proximity to a resonance. The difference between that and the numerical estimate is, at best, of the order of the estimate itself. In contrast, the second order analytical estimate manages to stay within $10 \%$ of the numerical value demonstrating the need for such high order theories. It is interesting to note that the observed deviations from the first order approximation are sufficient to produce a substantial lag (at least several tens of degrees) in the orbital longitude of a satellite on a $1 / 2$ day orbit around a giant planet over a period of a few years. In order to discover whether such mechanisms can account for observable variations in the properties of actual satellite systems we intend to extend our investigation to existing small satellite configurations in the near future.

\section{References}

Bosh, A. S. and Rivkin, A. S.: 1996, 'Observations of Satur's inner satellites during the May 1995 ring-plane crossing', Science, 272, 518-521.

Christou, A. A.: 1998, 'An investigation of secular perturbations in planetary and satellite systems', Ph. D. Thesis, University of London.

Christou, A. A. and Murray, C. D.: 1998, 'The effect of near-resonances on the secular precession of close orbits', Mon. Not. Royal Astron. Soc., submitted.

Levison, H. F. and Duncan, M. J.: 1994, 'The long-term dynamical behavior of short-period comets', Icarus, 108, 18-36.

Roddier C., Roddier F., Graves J. E., Guyon, O. and Northcott M. J.: 1998, 'Satellites of Neptune', IAU Circ., 6987.

Šidlichovský, M. and Nesvorný, D.: 1997, 'Frequency Modified Fourier Transform and its application to asteroids', Cel. Mech. Dyn. Astron., 65, pp. 137-149. 